\title{
Unsaturated Phosphazenes as Co-Solvents for Lithium-Ion Battery Electrolytes
}

Mason K. Harrup, Harry W. Rollins*, David K. Jamison, Eric J. Dufek, Kevin L. Gering, Thomas A. Luther

Idaho National Laboratory, P.O. Box 1625, Idaho Falls, ID 83415, USA

This paper covers the synthesis and use of a related family of cyclic phosphazene solvents containing terminal unsaturations that are being considered as electrolyte additives in lithium-ion cells. A brief synopsis on the synthesis and purification of these compounds is given. Data will be presented that covers physical and chemical properties of the phosphazenes as well as the properties when blended at various levels with representative organic carbonate baseline solvents. Electrochemical cycling data will be presented using commercially available electrode couples as well as studies focusing on cell performance at early lifetime as well as after repeated cycles. Conclusions regarding the effect of the unsaturated phosphazene compounds and their interaction with various alkyl carbonates, and their effect on cell performance will be presented.

*Corresponding author: Tel. +1 208526 4066, email harry.rollins@inl.gov 


\section{Introduction}

Lithium ion batteries are currently in widespread use for a broad range of mobile power needs. However, in order to move lithium ion technology into long-term larger format applications, such as vehicle technologies and stationary power, there still remain significant challenges that must be addressed. Perhaps the most urgent of these issues is safety concerns with the electrolyte. As most commercial electrolytes employ $\mathrm{LiPF}_{6}$ as the lithium salt dissolved in a mixture of organic carbonates and/or esters [1] this mix brings several intrinsic problems. These solvents are highly volatile as well as highly flammable. Also, they and their combustion products can be toxic. This presents a serious safety problem in situations where the battery is under abuse or if one (or more) of the individual cells in a pack design begins a thermal runaway, leading to propagation to the other cells. While this concern is partially mitigated in small single cell power sources such as those that power small electronics, the problem rapidly grows in magnitude as battery sizes and the numbers of individual cells in a pack grow. In order to have meaningful commercial adoption of such larger format batteries, this problem must be addressed.

The search for alternative solvents to serve as replacements for the current generation of alkyl carbonate-based solvents has received much attention in recent years. This is important not only from a safety standpoint but from a performance standpoint as the alkyl carbonates are known to have poor oxidative stability above 4.7-4.8 V [2]. Approaches to address these issues under study include the use of sulfones [3] and most recently ionic liquids [4, 5]. However it may be some time into the future before carbonates and esters may be entirely replaced. As a practical interim strategy, improvements in the performance and safety record of the current generation of electrolytes may be realized through the use of additives [6]. Additives have been the subject of research since the early 1990's [7] and continue to enjoy ever-increasing interest, especially in 
the past few years. Additive research has taken several forms to address various challenges in lithium ion batteries. Some have employed additives to improve solid electrolyte interphase (SEI) formation [8], overcharge protection [9], and flammability suppression [10]. Others have employed additives not as co-solvents but rather through the use of novel salts to achieve improved thermal stability [11]. Some of the classes of compounds originally investigated as complete replacements for the carbonates are undergoing a resurgence by employing them as cosolvents, such as ionic liquids [12].

There has been interest in the use of inorganic compounds for this purpose, particularly with the use of phosphorus compounds. Recent studies have employed phosphates as SEI improving additives [13], phosphites as additives [14], as well as phosphorus-nitrogen compounds to improve safety [15]. Previous work from our laboratories has shown that cyclic phosphazenes continue to exhibit real promise to improve both the safety and performance of conventional electrolytes [16-19]. This work reports on the behavior of novel liquid unsaturated phosphazenebased co-solvents for lithium ion batteries. This new class of phosphazene derivatives was chosen for study as it is well known that the incorporation of unsaturated moieties aids in SEI formation and subsequent battery performance [8].

\section{Experimental}

\subsection{Instrumentation}

All NMR analyses were performed on a Bruker Ascend Avance III $600 \mathrm{MHz}$ spectrometer. Viscosity measurements were performed using a Cambridge DL-4100 (falling bob) viscometer, in an argon glovebox, the data presented is the average of 10 individual measurements. Conductivity measurements were performed using a TOA CM-30R conductivity meter in an 
argon glovebox, the data presented is the average of 10 individual measurements. Flash point determinations were performed using a Setaflash 82000-0 (electric ignition) using a ramp determination method, the data reported is the average of 3 individual determinations, all with fresh sample. Vapor pressure measurements were made on a Grabner Instruments Minivap VPXpert. Trace water determinations were performed on a Mettler Toledo C30 Karl Fischer coulometer (in an argon glovebox), the data presented is the average of 3 individual measurements. Electrochemical measurements were made on a Solaratron SI 1260 impedance/phase gain analyzer with a Solaratron SI 1287 electrochemical interface. Coin cells (type 2032) were tested using a Maccor Series 4000 5V/5A full range tester. Thermal stability experiments were run in an ESPEC BTU133 thermal chamber.

\subsection{Reagents}

Hexachlorocyclotriphosphazene was obtained from Molport.com and was purified via sublimation immediately prior to use. All pendant groups were obtained from Aldrich Chemical Company, allyl alcohol was distilled and stored in Schenk bulb containers prior to use.

Isopropanol and ethanol were obtained as anhydrous grade and used as received. Sodium metal was obtained from Aldrich Chemical Company and the kerosene was rinsed off with hexanes and blotted dry with a laboratory wipe before use. Sodium hydride (95\%, dry) was obtained from Aldrich Chemical Company and used as received. 1,4-Dioxane was obtained from Aldrich Chemical Company as anhydrous grade and used as received. Dichloromethane was obtained from Aldrich Chemical Company and used as received. Nanopure water $(18.4 \mathrm{M} \Omega \mathrm{cm})$ was generated in the laboratory using a Barnstead NANOpure II water purification system. Ethylene 
carbonate, ethyl methyl carbonate, diethylene carbonate, and lithium hexafluorophosphate were all obtained as battery grade reagents from Kishida Chemical Co. and used as received.

\subsection{Phosphazene synthesis}

Synthesis of all of the phosphazenes followed a similar synthetic pathway employing Schlenk techniques. The specific formulation described here is for the synthesis of AL-6. Sodium hydride $(5.33 \mathrm{~g}, 222 \mathrm{mmol})$ was placed into an oven dried $2 \mathrm{~L}$ three-neck flask fitted with a reflux condenser, a dry nitrogen inlet, and a septum stopper. The dry nitrogen outlet issued from the top of the reflux condenser into an oil bubbler apparatus charged with approximately two inches of silicon oil. 1,4-Dioxane $(700 \mathrm{ml})$ was cannulated into the flask and the reaction was stirred using a magnetic stirbar. Freshly distilled allyl alcohol $(11.7 \mathrm{~mL}, 222 \mathrm{mmol})$ was cannulated into the reaction vessel and the reaction, which forms an allyl alkoxide solution, was allowed to proceed at room temperature overnight. In a separate $1 \mathrm{~L}$ three-neck flask outfitted as the first flask was placed sodium metal. (5.95g, $222 \mathrm{mmol})$ 1,4-Dioxane (350ml) was cannulated into the flask followed by addition of anhydrous ethanol $(16.0 \mathrm{~mL}, 274 \mathrm{mmol})$ and the reaction was stirred using a magnetic stirbar. The reaction was brought to a gentle reflux and allowed to proceed overnight. Hexachlorocyclotriphosphazene $(25.0 \mathrm{~g}, 72 \mathrm{mmol})$ was placed in a $500 \mathrm{ml}$ oven dried round bottomed flask fitted with a septum stopper. 1,4-Dioxane (200 ml) was cannulated in and the solution stirred magnetically. After dissolution was complete, the trimer solution was cannulated into the allyl alkoxide reaction solution. The reaction was brought to a

gentle reflux and reaction progress was monitored by ${ }^{31} \mathrm{P}$ NMR until it was determined that the reaction was complete, which took approximately two hours. When the ethoxide solution was determined to be complete evidenced by complete consumption of the sodium metal, that 
reaction mixture was added to the main reaction and the mix heated to a gentle reflux for two hours. Completion of the reaction was confirmed by ${ }^{31} \mathrm{P}$ NMR and the reaction was cooled to room temperature. The excess nucleophile was quenched with $250 \mathrm{ml}$ of water and brought to neutral $\mathrm{pH}$ with $2.0 \mathrm{M} \mathrm{HCl}$. The volume of the reaction mixture was reduced on a rotary evaporator to a final volume of $300 \mathrm{ml}$. This solution was transferred to a $2 \mathrm{~L}$ separatory funnel and $500 \mathrm{ml}$ of dichloromethane was added, forming two phases. These were separated and the dichloromethane phase was retained. This was returned to the separatory funnel where it was subsequently washed seven times with fresh aliquots of nanopure water (500 $\mathrm{ml} \mathrm{each).} \mathrm{The} \mathrm{final}$ product was obtained by the removal of the dichloromethane solvent by rotary evaporation to yield a clear, slightly yellow oil. The oil was dried in a vacuum/argon oven at $80{ }^{\circ} \mathrm{C}, 25 \mathrm{mTorr}$ Ar for a minimum of $72 \mathrm{~h}$. Yield was $25.2 \mathrm{~g}, 78 \% .{ }^{31} \mathrm{P}$ NMR: $17.5 \mathrm{ppm}(\mathrm{m})$.

\subsection{Coin cell build and testing}

Full cell evaluation of each of the electrolyte chemistries of interest was performed using CR 2032-type coin cells. Prior to building cells all non-separator components were dried overnight at $90^{\circ} \mathrm{C}$ under vacuum. Separators were dried overnight at $60^{\circ} \mathrm{C}$ under vacuum overnight. All cells were built under an Ar atmosphere with oxygen and water content below $0.1 \mathrm{ppm}$. Cells were constructed using a G8 (Conoco-Phillips) graphite based anode and a proprietary blended $\mathrm{Li}_{1.06} \mathrm{Mn}_{1.94} \mathrm{O}_{4}+\mathrm{Li}_{1.1} \mathrm{Ni}_{0.33} \mathrm{Mn}_{0.33} \mathrm{Co}_{0.33} \mathrm{O}_{2}$ cathode. Both the anode and cathode materials were calendared to a uniform thickness prior to use; the geometric area of each electrode was 1.43 $\mathrm{cm}^{2}$. Consistent loading of the active materials onto the current collectors was confirmed by weighing same-area electrode samples, which showed very close agreement in weight. A Celgard 2325 separator $\left(1.58 \mathrm{~cm}^{2}\right)$ was used to separate the electrodes. During the build process both the anode and cathode were flooded with electrolyte to ensure uniform electrolyte 
distribution within each electrode. The nominal rated capacity of the materials (cell basis) is 3.65 $\mathrm{mAh}$ per coin cell or $2.55 \mathrm{mAh} \mathrm{cm}^{-2}$. To ensure reproducibility all cell formulations were evaluated in triplicate. Following construction, stainless steel tabs were spot welded onto the positive and negative terminals of the cell respectively prior to mounting in a cell holder which was designed and built in house. The holder maintained the cells in a horizontal position with only slight tilting in any one direction. Within the holder, each cell was spaced a minimum of 5 $\mathrm{cm}$ from its nearest neighbor with open space on all sides. This orientation is preferred to minimize potential pooling of electrolyte within the cell and to provide a uniform thermal profile from cell to cell. Full cell testing was performed using a 4000 Series Maccor test system that accomplished up-front formation cycling based on C/10 constant-current (CC) conditions, and other CC conditions to determine aging behavior and sensitivity of cell performance to rate. Typical cycling occurred between 3.0 and $4.2 \mathrm{~V}$ at various cycling rates. Cells were maintained at a constant temperature $\left(30^{\circ} \mathrm{C}\right)$ in environmental chambers (Tenney or Espec) during testing. Prior to initiating testing protocol each Maccor channel was calibrated. Once built individual cells were allowed to equilibrate to $30^{\circ} \mathrm{C}$ over $2 \mathrm{~h}$ in the environmental chambers. Electrochemical impedance spectroscopy (EIS) data was acquired using a Solartron SI 1287 Electrochemical Interface and a Solartron SI 1260 Impedance/Gain-phase analyzer. EIS were acquired at open circuit after discharging the cell to $3.75 \mathrm{~V}$ and then charging to $3.9 \mathrm{~V}$ and were taken at frequencies ranging from 0.1 to $100000 \mathrm{~Hz}$ with $20 \mathrm{mV}$ amplitude.

\section{Results and Discussion}

\subsection{Phosphazene structures}


The AL series of phosphazenes is constructed from light alcohols, some that are saturated and some that contain terminal unsaturations in a logical related series to better understand the influence of the unsaturated groups on the behavior as a co-solvent in the electrolytes. All employ identical trimeric phosphazene cores. Shown in Figure 1 is a representative member of the series, AL-7. The other members of this series are related derivatives, and Table 1 gives the pendant group distribution for each member of the series. Previous work has shown that even slight variations can exhibit significant differences on chemical and physical properties in a given phosphazene series [18], and part of this work is to investigate whether this observation holds valid for the unsaturated phosphazenes as well.

\subsection{Physical characterization of the phosphazene blends}

All phosphazenes were placed in an argon glovebox after sufficient purification was confirmed. This consisted of a halogen analysis employing ICP-MS techniques, a total acid analysis, and total water analysis employing Karl-Fisher microtitration. The acceptable threshold for purity was $<10 \mathrm{ppm}$ which is a complete non-detect [20]. Phosphazene:carbonate solvents were blended volumetrically, and subsequently salted to the proper $\mathrm{LiPF}_{6}$ concentrations in an argon glovebox. The electrolytes were then studied by a variety of physical analytical techniques to determine the effect of the phosphazenes on the baseline carbonates. The first of these measurements was to determine the total conductivity of the electrolyte blends. As shown in Figure 2, conductivity of the blends declines with the addition of increasing levels of phosphazene. For blend A, (EC:DEC blend) the initial conductivity is lower than that found for blend B (EC:EMC blend), hence the conductivity remains concomitantly lower at each stage of increasing phosphazene addition. There are two points of note in this characterization. First, the 
total percentage drop in conductivity is remarkably similar between the two blends, at about $43 \%$ for the highest phosphazene concentration. Second, all of the various formulations of phosphazene produce nearly identical effects on conductivity in both carbonate baselines. This is indicative that the phosphazene itself is of lower conductivity than the baseline carbonates, and that changes in the pendant group structure of the AL class have little effect on differences in conductivity.

The second characterization technique studied was the effect of phosphazene addition to the baseline formulations on viscosity. As shown in Figure 3, phosphazene addition did cause an increase in the viscosity of the blends. The increase in viscosity of both the baseline blends was nearly identical, increasing from about $4 \mathrm{cP}$ to $10 \mathrm{cP}$ for blends containing $30 \%$ phosphazene. Similarly, each of the AL formulations exhibited nearly identical viscosities at each loading level. This provides further evidence that these properties are being affected little by the nature of the pendant group structure. This in turn may be indicative that these physical properties are being dominated by the phosphazene core structure itself when substituted with short-chain unsaturated pendant groups.

The third characterization technique to be employed was a closed-cup flash point analysis. Unlike the other characterization techniques discussed in this paper, out of necessity it was not possible to conduct these measurements in the glovebox due to the need for atmospheric levels of oxygen. As shown in Figure 4, the flash point of the electrolyte formulations fairly linearly rises with increasing levels of phosphazene addition. At 30\% phosphazene the flash point increases by approximately $20 \%$ over the baseline electrolyte for both blends A and B. As was found in the previous characterizations, there were little differences in the behavior of the various phosphazene formulations on the flash points observed. Also as in the other 
characterization techniques, there was little difference in the increase in flash point between blends A and B. This provides more weight to the argument that changes in the pendant group structure of the AL class have little effect on the properties of the blended electrolytes.

The fourth characterization technique employed was a study of the vapor pressure as a function of temperature. The results of this study are presented in Figure 5. All of the phosphazene data presented employs a $30 \%$ loading level. The addition of the phosphazenes has the expected effect of lowering the vapor pressure of the electrolyte blends. As seen in the other characterization techniques, there appears to be little difference in the behavior of the blends between members of the AL series phosphazenes. This serves to confirm the arguments presented above that changes in the composition of the AL phosphazenes in this series has little impact in the physical properties of the blends.

\subsection{Cycling performance}

All of the electrolyte blends were employed to build 2032-type coin cells using the materials described in the Experimental Section above. The performance of these cells was investigated and the cells were cycled for an extended period at a $\mathrm{C} / 3$ discharge rate. Both baselines A and $\mathrm{B}$ were investigated independently. Discharge capacities were recorded after each cycle and a reference performance test (RPT) which consisted of cycling the cells at an assortment of rates $(\mathrm{C} / 10, \mathrm{C} / 3$ and $\mathrm{C} / 1)$ was conducted after each set of twenty $\mathrm{C} / 3$ cycles. Representative data for the AL-5 blends are presented in Figure 6 for the first 80 cycles. It is clear that with lower percentages of added phosphazene, performance at this discharge cycling rate is functionally identical to that observed in Baseline A. However, at higher loading levels there is a marked decrease in the performance as compared to the baseline. This is to be expected, as the 
viscosities of the formulations are higher, while simultaneously possessing a lower conductivity. While these effects are not pronounced in lower loading levels, they become much more evident in the performance of the cells at higher loading levels. Also of note is the rate of decline in the capacity as a function of cycle rate. While at early cycle times the baseline electrolyte possesses slightly higher capacity, the rate of capacity fade without the phosphazene is actually greater, leading to performance crossover at cycle thirty. Similarly, the rate of increase in capacity fade is more pronounced at late cycle times when using the baseline electrolyte. This is indicative that the phosphazene has a positive influence on the stability of the cell chemistry when used at levels between 5-10\%. Previous reports have shown that similar phosphazene additives which incorporate fluorinated pendants improve SEI stability [21]. Surprisingly, this trend in capacity fade rates is reversed in baseline B. It is the electrolytes containing low levels of phosphazene that possess the higher initial capacity at early cycle times, but exhibits a more rapid capacity fade with continued cycling. As the only significant difference in the two systems is one constituent of the alkyl carbonate solvents (A has DEC, B has EMC), this suggests that the phosphazene AL-5 has a less favorable interaction with EMC as compared to DEC.

A similar behavior is observed with the other phosphazenes, AL-6 and AL-7 as shown in Figures 7 and 8, respectively. In baseline A, electrolytes with lower loading levels of phosphazene improve the baseline performance, both in terms of total discharge capacity as well as exhibiting a slower rate of capacity fade. This is indicative that the phosphazenes, regardless of specific formulation (AL-5, AL-6, AL-7) form a more stable electrolyte when used in conjunction with DEC. In baseline B (EC-EMC) similar results were observed with AL-6 and AL-7 as to those found with baseline A, in contrast to what was observed for the AL-5. As the AL-5 is the phosphazene with the lowest number of unsaturated groups, it may be that this lower 
level of unsaturations is ineffective at providing the benefits observed in formulations that possess a higher total percentage of unsaturated groups.

\subsection{Initial capacities}

To further investigate the manner in which the phosphazenes alter the electrolyte chemistry, a comparison of the initial rates after formation was conducted and is given in Figure 9. Two expected trends are readily observed. First, the discharge capacities are higher for the slower cycling rates in all cases. Second, higher loading levels of phosphazene produce a pervasive trend of lower discharge capacities. This is expected given the lower conductivities observed for the higher phosphazene loading levels (Figure 2). However there are differences in the observed behavior between the members of the AL series. In baseline A, AL-6 has a greater reduction in capacity as a function of loading, i.e. there is a larger detriment to capacity as loading increases. Similarly this trend is continued for AL-7 and AL-5, with improvement in performance as the number of unsaturations decreases. These trends generally continue in baseline B, but as noted in other cell performance metrics, there is a greater variability in the initial capacities in baseline $\mathrm{B}$, suggesting a more significant interaction between these phosphazenes and EMC as compared to DEC.

\subsection{Electrochemical Impedance Spectroscopy}

A study was conducted to probe the effects of the phosphazene at later cycle times, and to correlate that to behavior at early cycle times to gain a better understanding of how the unsaturated phosphazenes affect the electrolyte chemistry. EIS measurements were made after 50 cycles (at $\mathrm{C} / 3$ discharge) and are given in Figures 10 and 11. The semicircles seen in the 
Nyquist plots represent mechanistic processes that influence lithium transport between the free electrolyte region (high frequencies, toward left side of plots) and the solid state domain (Warburg region, right-most plot attribute), that is, the semicircles capture primarily interfacial attributes involving the charge transfer process, influence of SEI traits, and lithium de-solvation. It is within this bracketed region we focus our attention for identifying unique interfacial features related to the transport of lithium ions through the electrolyte-passivated surface. A comparison between various loading levels for a representative phosphazene (AL-5, Figure 10) shows an unsurprising trend. After cycling, the impedance associated with bulk transport in the electrolyte remains very similar to that observed in the baseline, indicating that the bulk solution properties of the electrolytes is little changed. However the impedance associated with the interfacial charge transfer process is markedly different from the baseline. This is to be expected as the terminal unsaturations on the phosphazenes are known to be more reactive than the alkyl carbonates themselves. Further reaction between the phosphazene and the SEI layers at the electrode interface would lead to higher impedance over time and with increased cycling. Further, the general trend observed is that electrolytes that contain more phosphazene show higher impedance. This is understandable given that there are more unsaturated groups that could participate in degradation reactions. Whether this higher impedance is due to a simple thickening of the SEI, or to a new SEI component that is less ionically conductive cannot be determined from these experiments, but it is likely that some combinations of these two mechanisms is at work in these systems. As seen in Figure 11, a comparison between the phosphazenes again shows higher impedance only for the lower frequency semi-circle. All three impedances are functionally equivalent in baseline A, but are markedly different in baseline B. This adds weight to the argument made in the previous sections that the phosphazenes have a 
stronger interaction with the EMC than observed for the DEC. Curiously, even though the unsaturations on the phosphazene are the most reactive species in the electrolyte formulations, in baseline $\mathrm{B}$ the phosphazenes with the most unsaturations show the lowest impedance, with a linear trend towards higher impedance as unsaturation amounts decline. This may be due to some beneficial effect that the unsaturations have on cell performance.

\section{Conclusions}

From the data presented, several conclusions can be drawn. First, the physical properties of the blends are nearly identical between the three phosphazenes studied, indicating that the number of unsaturations in the phosphazenes have little impact on the physical characteristics of the resultant electrolyte formulations. Second, at lower loading levels of phosphazene, discharge performance is improved over both baseline solvents. At higher loading levels, performance is reduced as compared to the baselines, principally due to the increases in viscosity and lowered conductivity. Third, the total number of unsaturations per phosphazene has a small but noticeable impact on cell performance, with a higher degree of unsaturations generally exhibiting poorer performance. Also, the choice of baseline electrolyte does determine the eventual fate and benefit of the phosphazene additives, for example, as seen in the decrease in capacity loss within the Baseline A blends with lower amounts of additives. Finally, many of the beneficial effects demonstrated by this family of phosphazenes are likely due to the phosphazenic core as demonstrated in other phosphazene work from our labs. The incorporation of unsaturations in the phosphazene has a positive effect at lower levels, but loses much of these benefits at higher loading levels. Overall, phosphazene co-solvents continue to hold promise for improving battery 
safety and performance when incorporated into multi-solvent electrolyte systems for lithium ion batteries.

\section{Acknowledgments}

The authors gratefully acknowledge Peter Faguy and David Howell and the Office of Energy Efficiency and Renewable Energy, ABR Program within the United States Department of Energy. The authors also acknowledge support from the Idaho National Laboratory per contract DE-AC07-05ID14517. 


\section{References}

[1]. Advances in Lithium-Ion Batteries, W. A. Van Schalkwijk and B. Scrosati, Eds., Kluwer Academic, New York (2002).

[2]. L. Hu, Z. Zhang, K. Amine, J. Power Sources 236 (2013) 175-180.

[3]. Y. Watanabe, S. Kinoshita, S. Wada, K. Hoshino, H. Morimoto, S. Tobishima, J. Power Sources 179 (2008) 770-779.

[4]. A. Balducci, S. Jeong, G. Kim, S. Passerini, M. Winter, M. Schmuck, G. Appetecchi, R. Marcilla, D. Mecerreyes, V. Barsukov, V. Khomenko, I. Cantero, I. De Meatza, M. Holzapfel, N. Tran, J. Power Sources 196 (2011) 9719-9730.

[5]. Z. Wang, Y. Cai, T. Dong, S. Chen, X. Lu, Ionics 19 (2013) 887-894.

[6]. J. Goodenough and Y. Kim, Chem. Mater. 22 (2010) 587-603.

[7]. T. Hirai, I. Yoshimatsu, J. Yamaki, J. Electrochem. Soc. 141 (1994) 2300-2305.

[8]. C. Chang, S. Hsu, Y. Jung, C. Yang, J. Power Sources 196 (2011) 9605-9611.

[9]. Y.-G. Lee and J. Cho, Electrochim. Acta 52 (2007) 7404-7408.

[10]. S. Dalavi, M. Xu, B. Ravdel, L. Zhou, B. Lucht, J. Electrochem. Soc. 157 (2010) A1113-A1120.

[11]. M. Xu, L. Zhou, L. Hao, L. Xing, W. Li, B. Lucht, J. Power Sources 196 (2011) 6794-6801.

[12]. L. Lombardo, S. Brutti, M. A. Navarra, S. Panero, P. Reale, J. Power Sources 227 (2013) 8-14.

[13]. I.-J. Park, T.-H. Nam, J.-G. Kim, J. Power Sources 244 (2013) 122-128.

[14]. Z.D. Li, Y.C. Zhang, H.F. Xiang, X.H. Ma, Q.F. Yuan, Q.S. Wang, C.H. Chen, J. Power Sources 240 (2013) 471-475. 
[15]. B. Wu, F. Pei, Y. Wu, R. Mao, X. Ai, H. Yang, Y. Cao, J. Power Sources 227 (2013) 106-110.

[16]. M. K. Harrup, J. R. Delmastro, F. F. Stewart, T. A. Luther, Safe Battery Solvents, U.S. Patent 7,285,362 B2 (2007).

[17]. S. V. Sazhin, M. K. Harrup, K. L. Gering, J. Power Sources 196 (2011) 3433-3438.

[18]. M. K. Harrup, K. L. Gering, H. W. Rollins, S. V. Sazhin, M. T. Benson, D. K. Jamison, C. J. Michelbacher, T. A. Luther, ECS Trans. 41 (2012) 13-25.

[19]. H. W. Rollins, M. K. Harrup, E. J. Dufek, D. K. Jamison, S. V. Sazhin, K. L. Gering, D. L. Daubaras, J. Power Sources 263 (2014) 66-74.

[20]. The K-F titrator employed has a stated detection limit at or near $10 \mathrm{ppm}$ water. Only a non-detect was considered acceptable.

[21]. S. V. Sazhin, K. L. Gering, M. K. Harrup, H. W. Rollins, J. Electrochem. Soc. 161 (2014) A393-A402. 
Figure 1. Molecular structure of the phosphazene AL-7.

Figure 2. Conductivity as a function of phosphazene loading in two representative carbonate solvents.

Figure 3. Viscosity as a function of phosphazene loading in two representative carbonate solvents.

Figure 4. Flash point as a function of phosphazene loading in two representative carbonate solvents.

Figure 5. Vapor pressure as a function of temperature with $30 \%$ loading of the AL phosphazenes compared to the baseline electrolytes alone.

Figure 6. Discharge capacities of the electrolytes loaded with AL-5 at C/3 rate for the first 80 cycles. $\left(\right.$ Electrode area $=1.43 \mathrm{~cm}^{2}$ )

Figure 7. Discharge capacity of electrolytes loaded with various levels of AL-6 at C/3 rate for the first 80 cycles. (Electrode area $=1.43 \mathrm{~cm}^{2}$ )

Figure 8. Discharge capacity of electrolytes loaded with various levels of AL-7 at C/3 rate for the first 80 cycles. (Electrode area $=1.43 \mathrm{~cm}^{2}$ )

Figure 9. Initial discharge capacity of the cells in both baselines. Represented data is an average of three individual cells. (Electrode area $=1.43 \mathrm{~cm}^{2}$ )

Figure 10. EIS spectra of AL-5 at various loading levels.

Figure 11. A comparison of the EIS spectra between the three phosphazene co-solvents. 
Table 1. Pendant group distribution of the AL series of phosphazenes. 
Figures

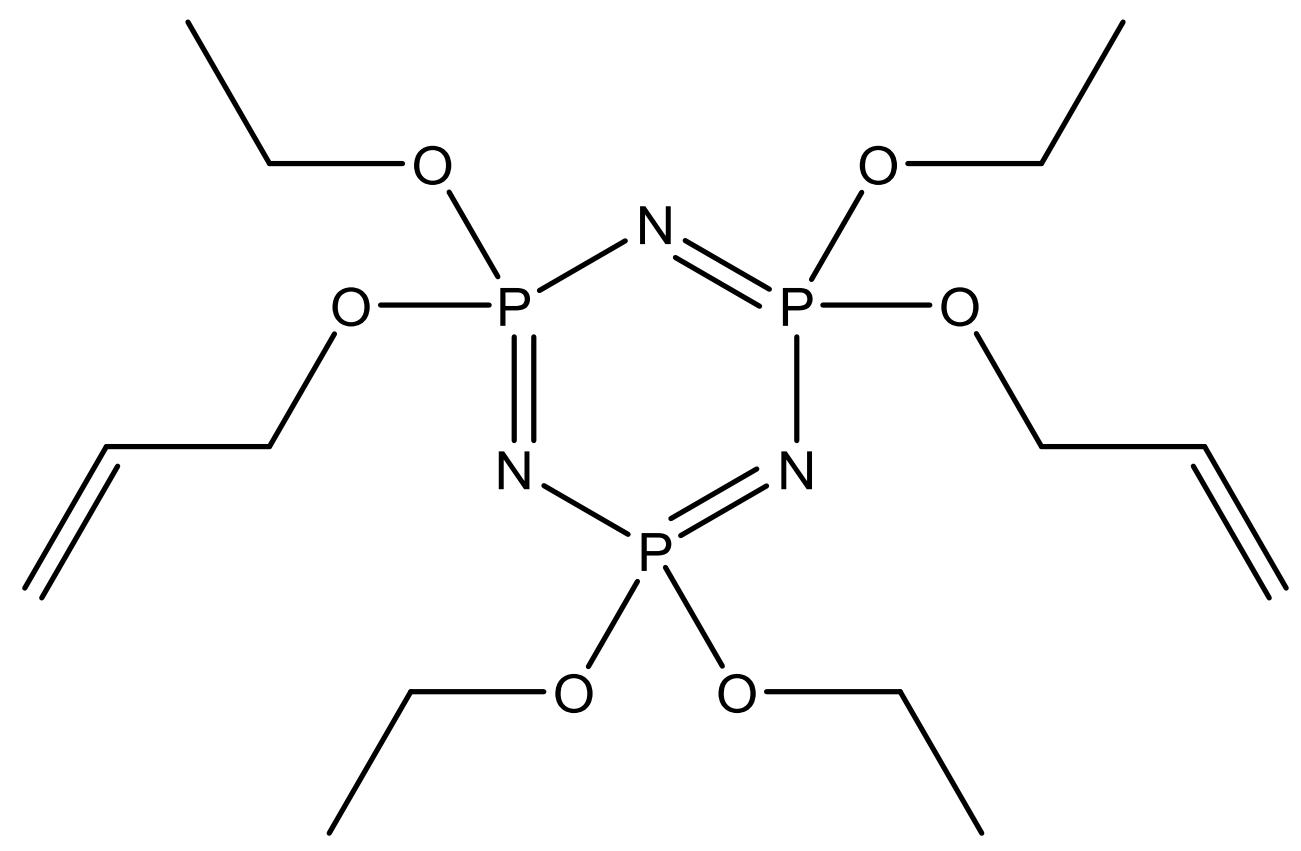

Figure 1. Molecular structure of the phosphazene AL-7. 

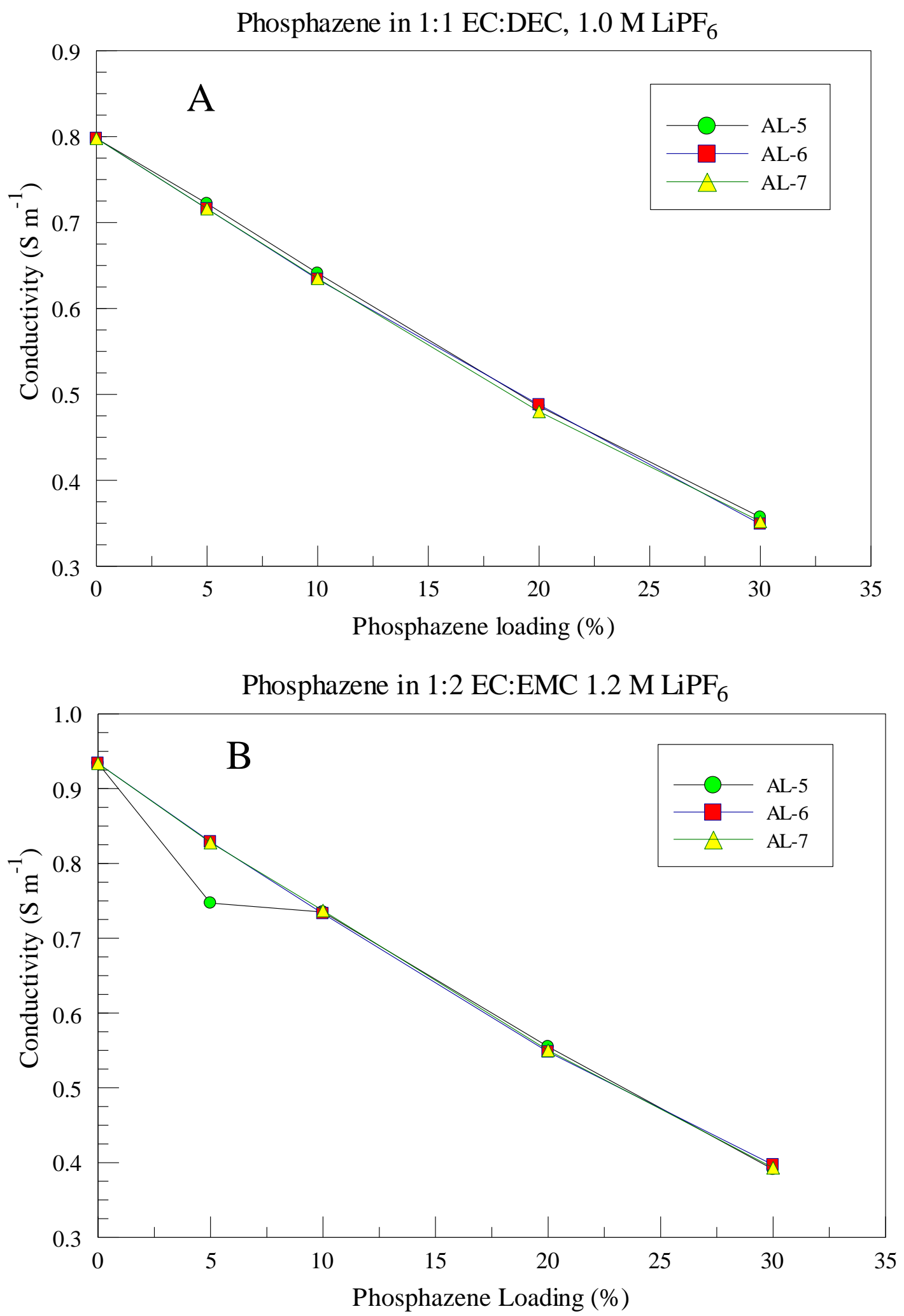

Figure 2. Conductivity as a function of phosphazene loading in two representative carbonate solvents. 

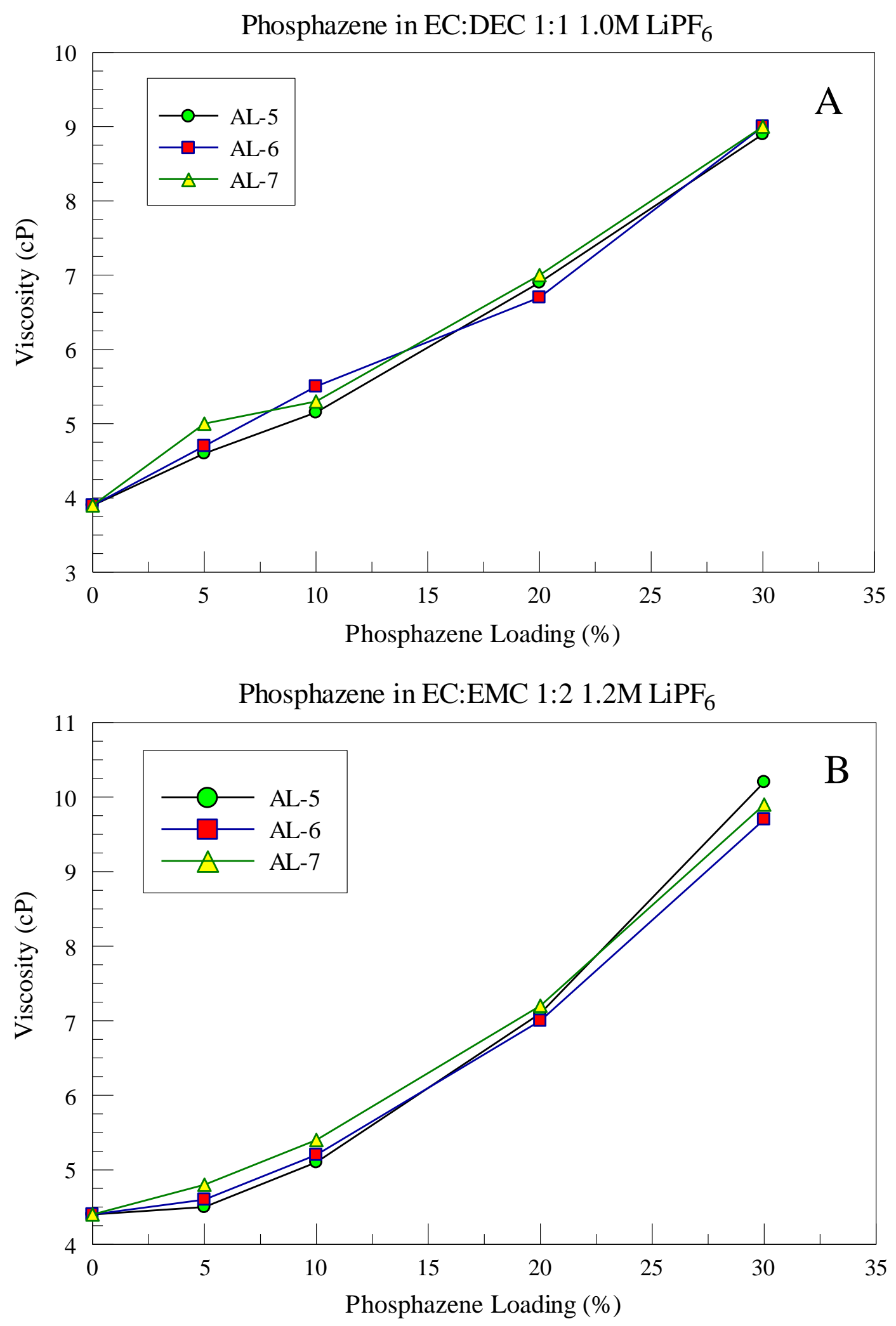

Figure 3. Viscosity as a function of phosphazene loading in two representative carbonate solvents. 
Phosphazene in EC:DEC 1:1 1.0M LiPF 6

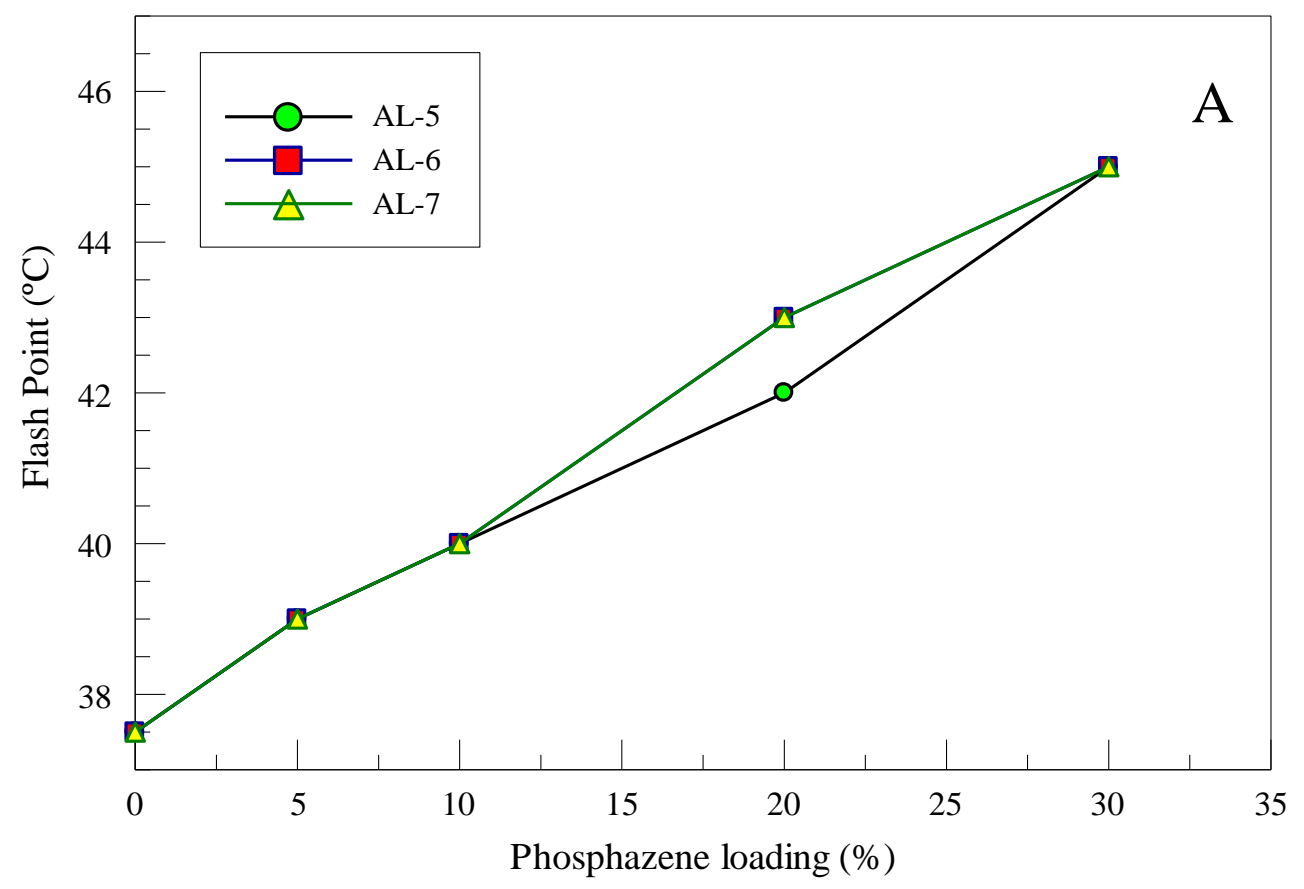

Phosphazene in EC:EMC 1:2 1.2M LiPF

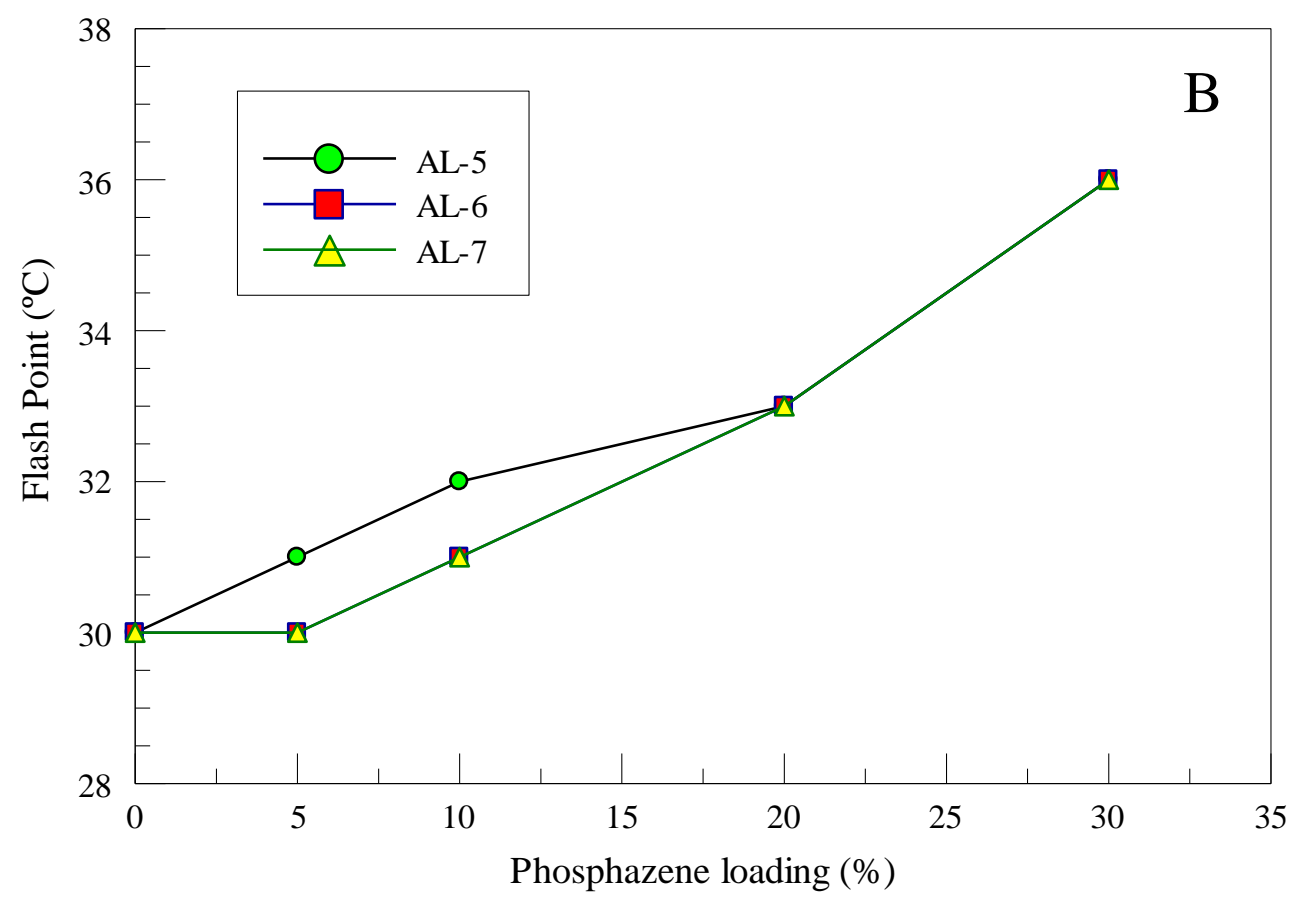

Figure 4. Flash point as a function of phosphazene loading in two representative carbonate solvents. 

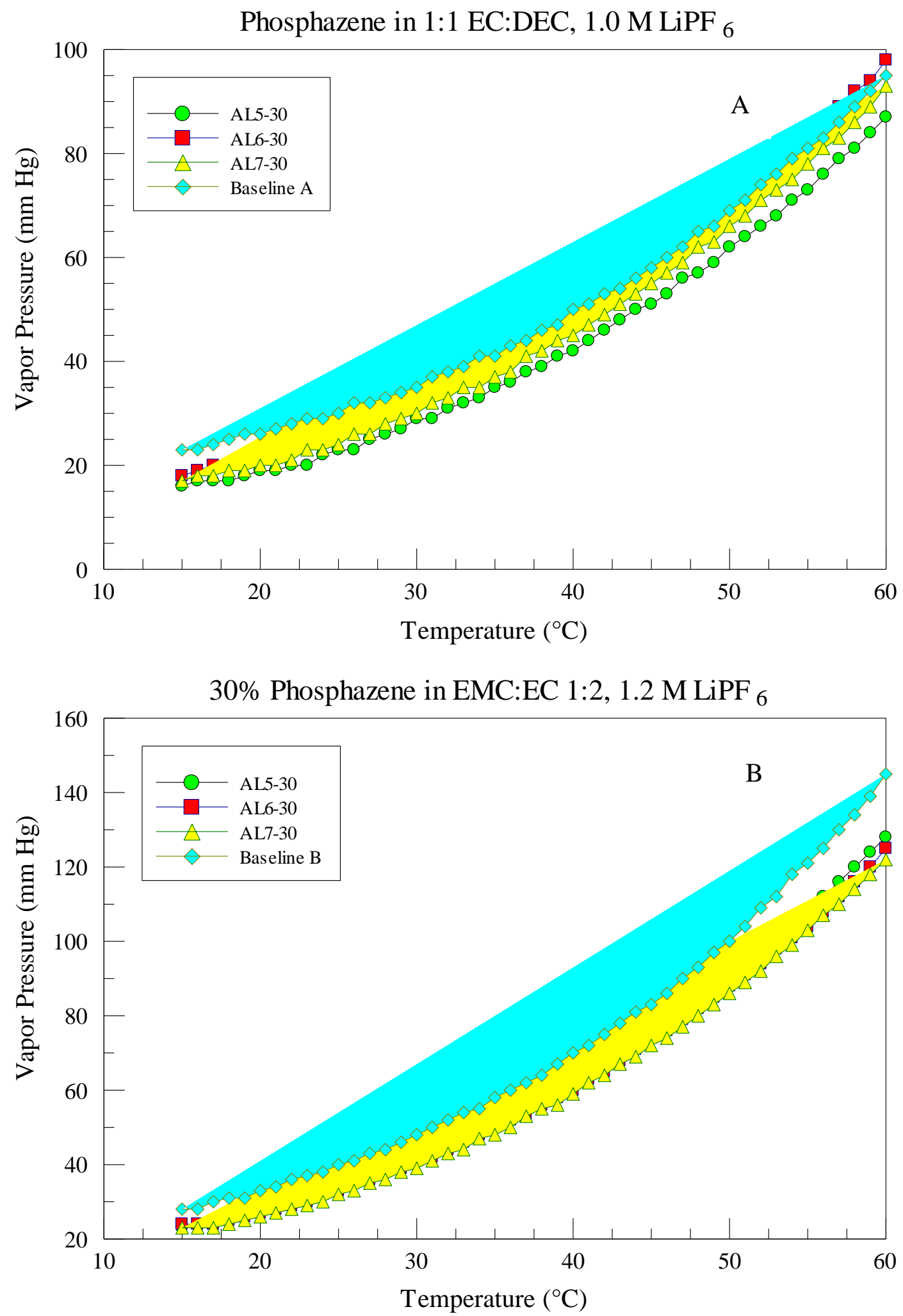

Figure 5. Vapor pressure as a function of temperature with $30 \%$ loading of the AL phosphazenes compared to the baseline electrolytes alone. 

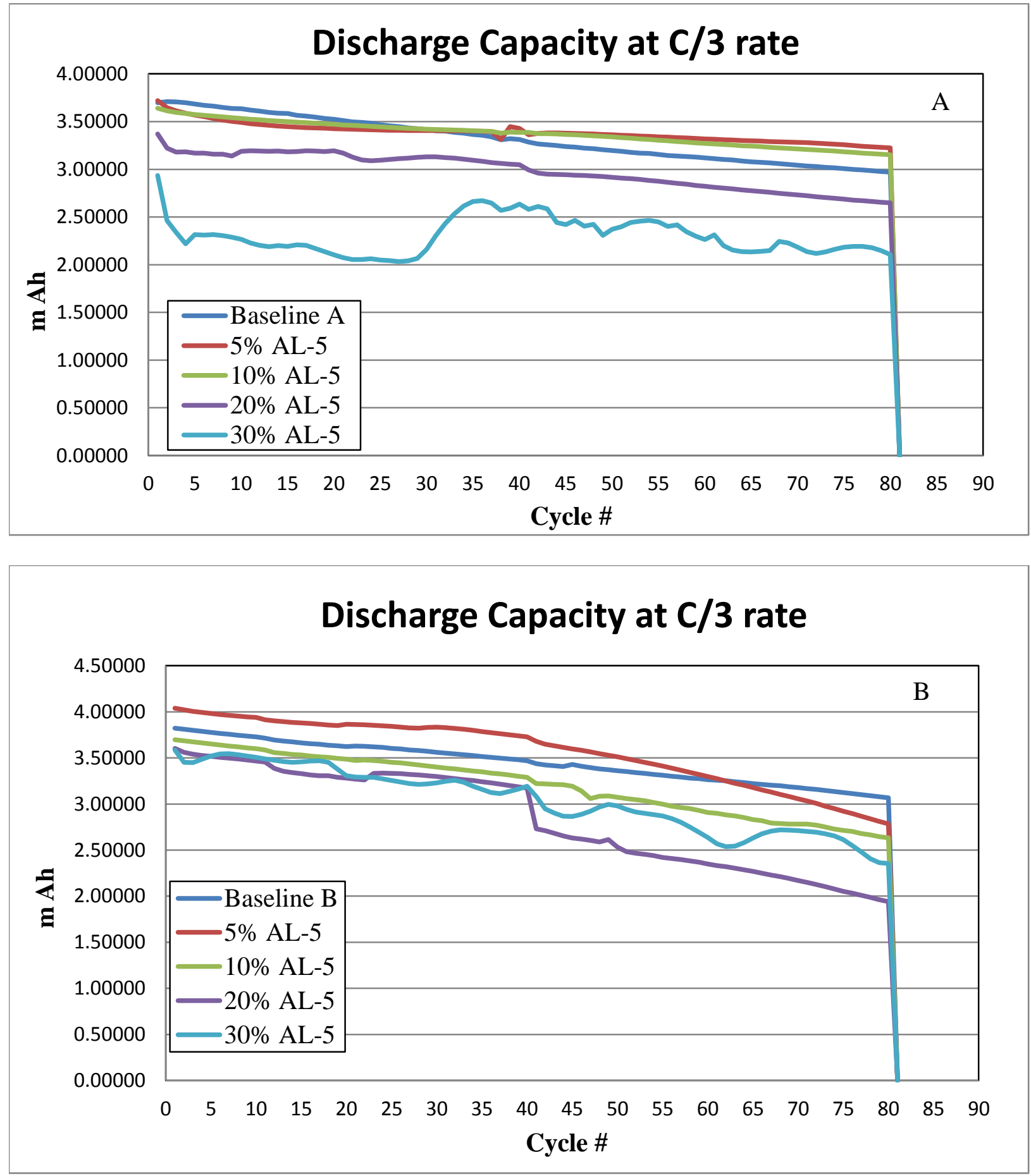

Figure 6. Discharge capacities of the electrolytes loaded with AL-5 at C/3 rate for the first 80 cycles. (Electrode area $=1.43 \mathrm{~cm}^{2}$ ) 

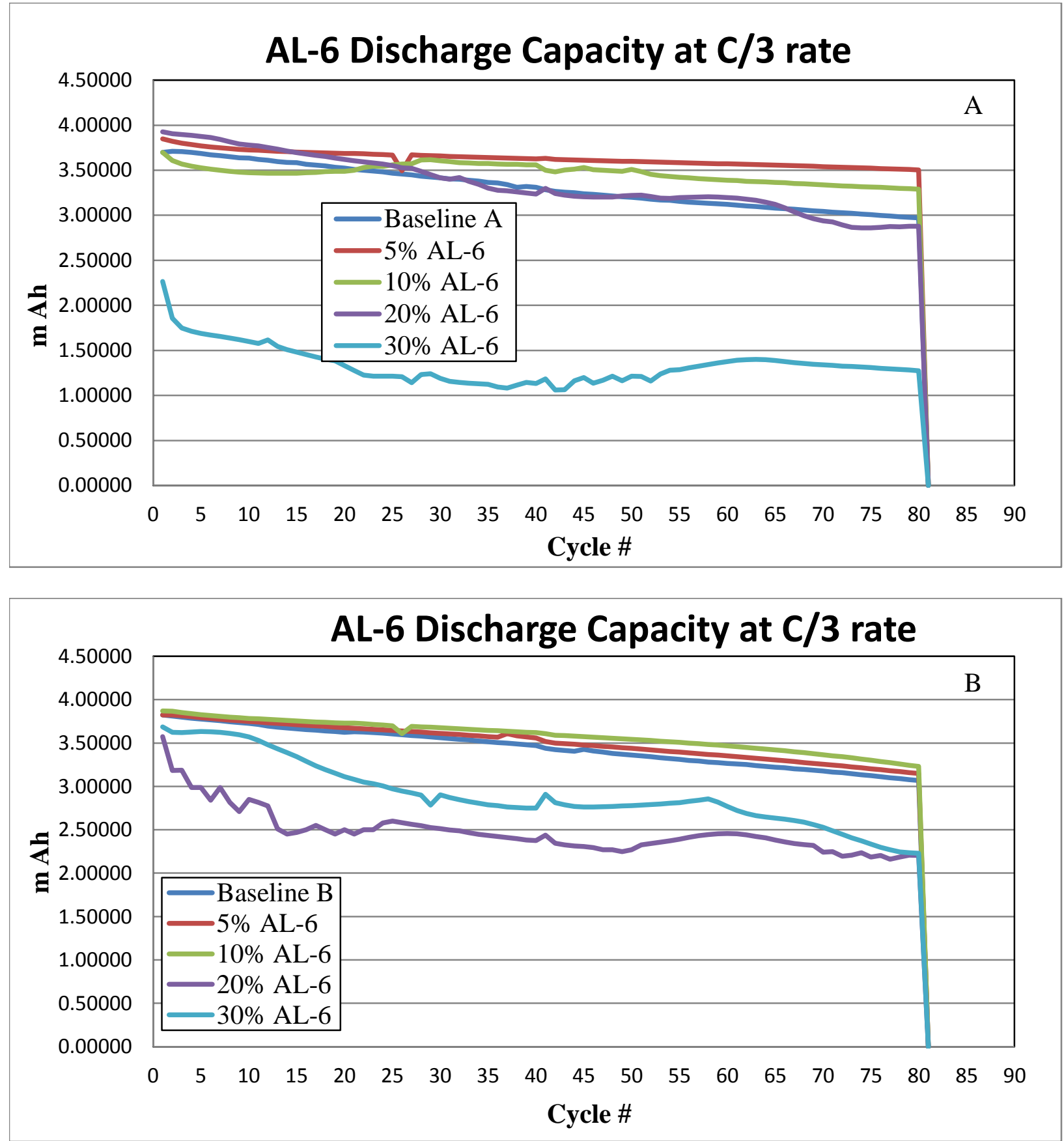

Figure 7. Discharge capacity of electrolytes loaded with various levels of AL-6 at C/3 rate for the first 80 cycles. (Electrode area $=1.43 \mathrm{~cm}^{2}$ ) 

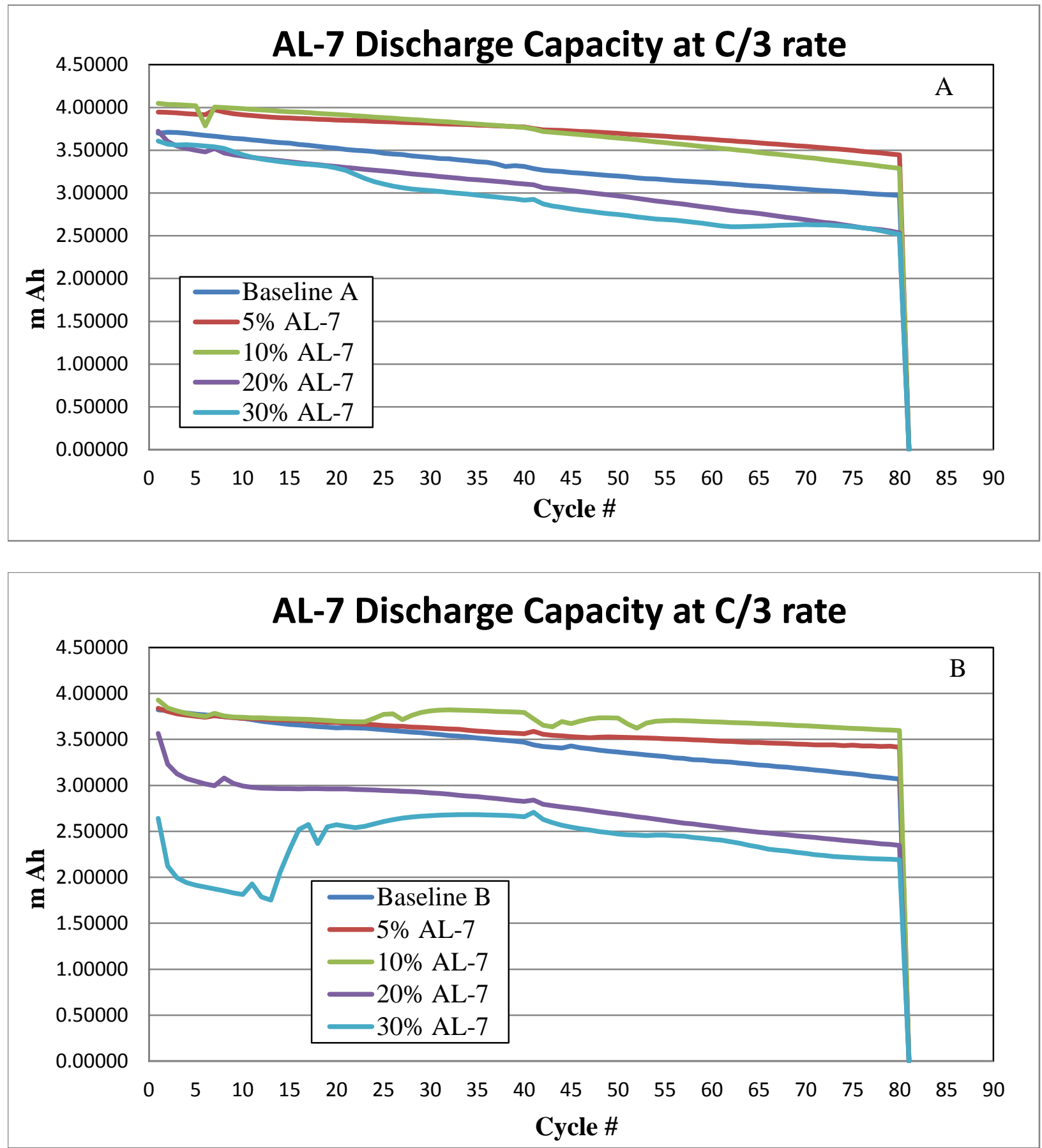

Figure 8. Discharge capacity of electrolytes loaded with various levels of AL-7 at C/3 rate for the first 80 cycles. (Electrode area $=1.43 \mathrm{~cm}^{2}$ ) 

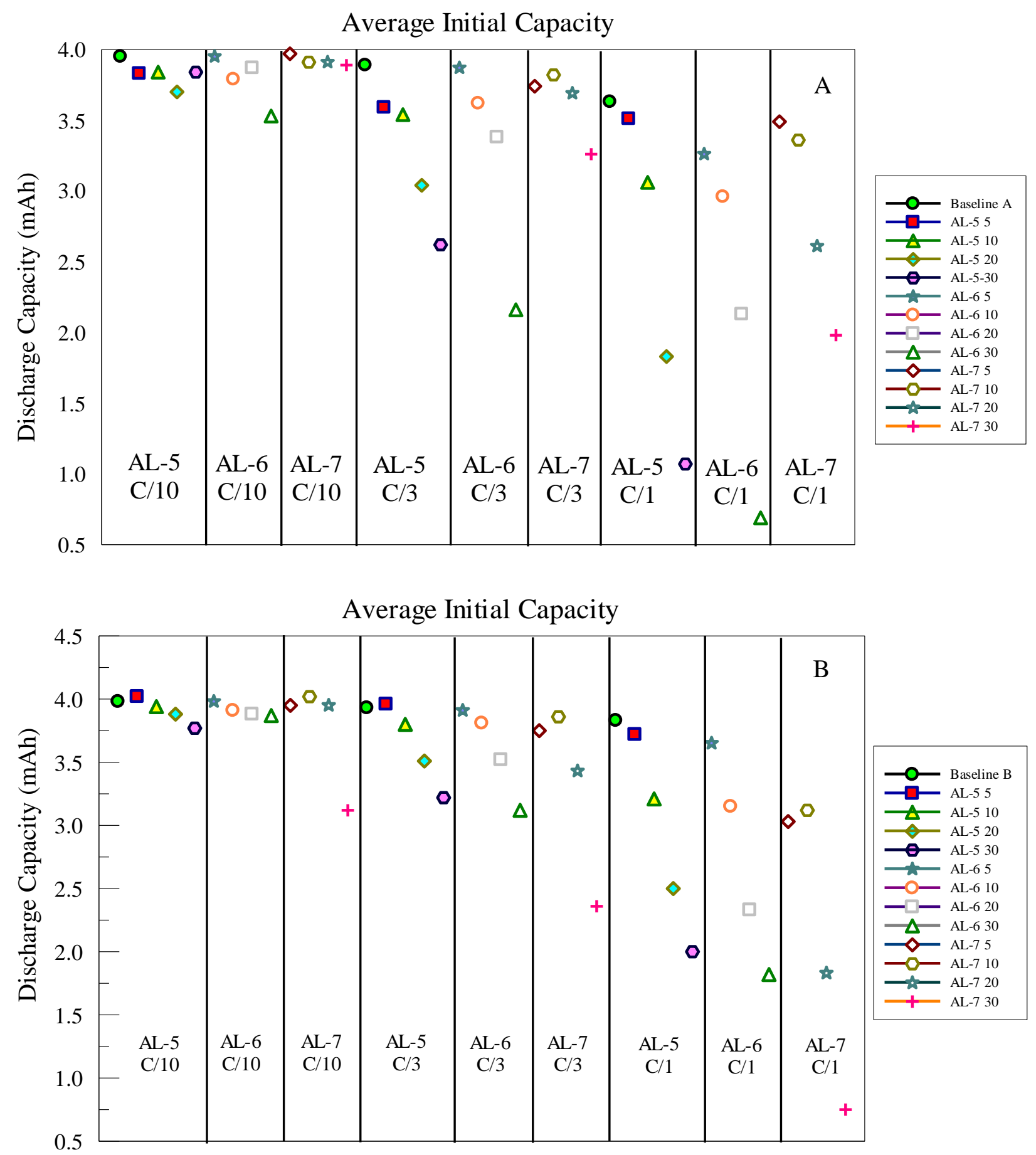

Figure 9. Initial discharge capacity of the cells in both baselines. Represented data is an average of three individual cells. (Electrode area $=1.43 \mathrm{~cm}^{2}$ ) 

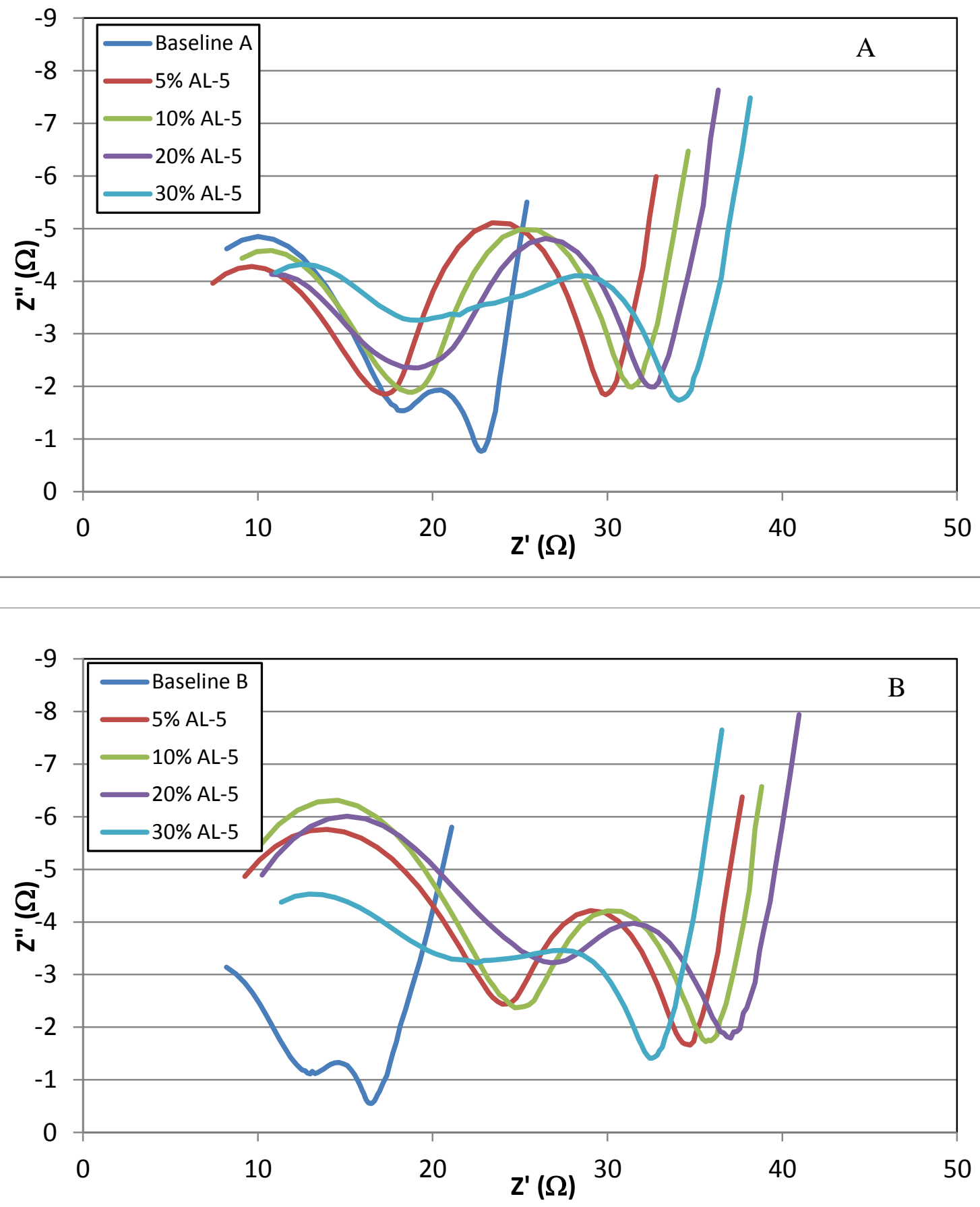

Figure 10. EIS spectra of AL-5 at various loading levels. 

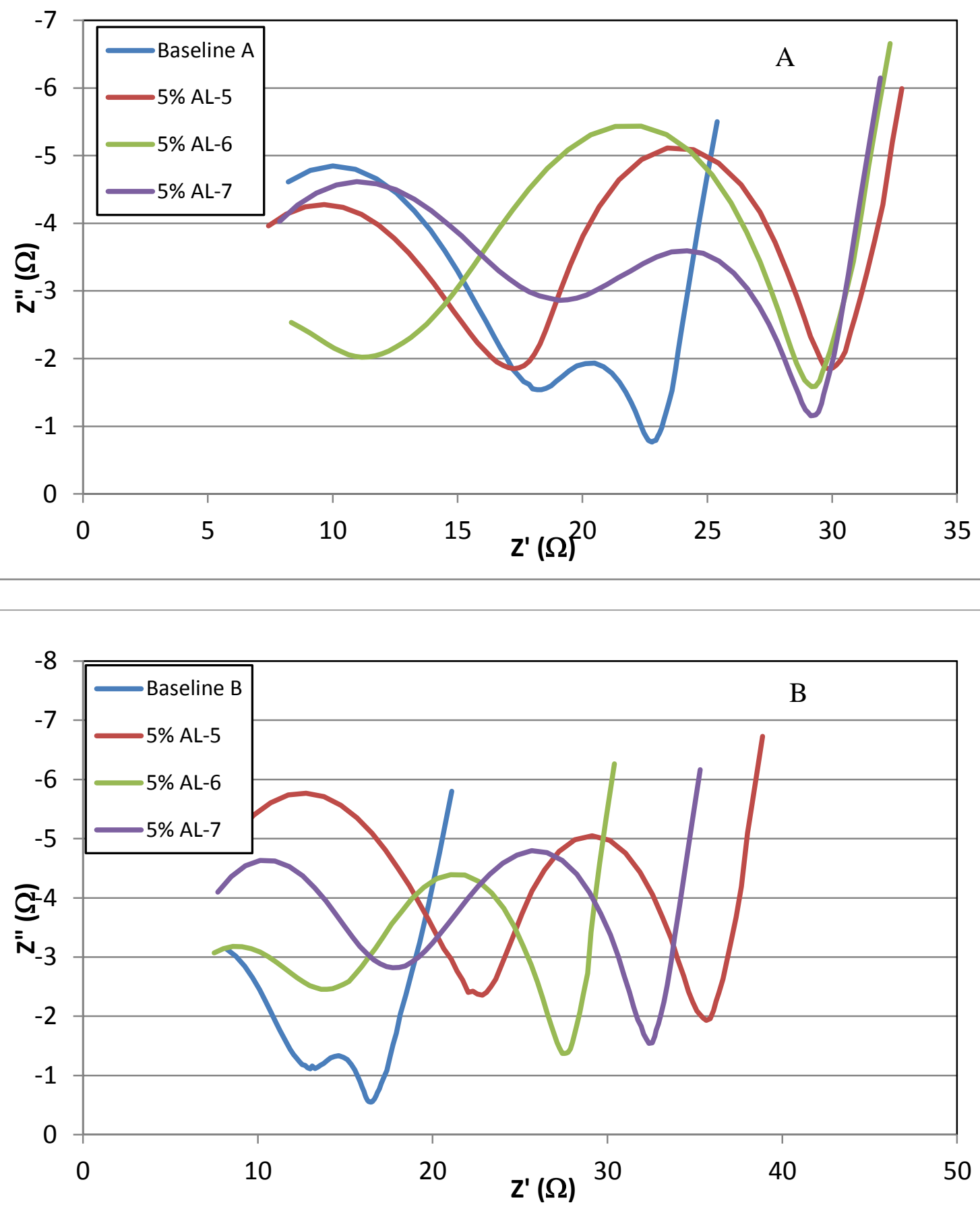

Figure 11. A comparison of the EIS spectra between the three phosphazene co-solvents. 


\section{Tables}

Table 1.

\begin{tabular}{|c|c|c|}
\hline Designation & 1 & 5 \\
\hline $\mathrm{AL}-5$ & 3 & 3 \\
\hline $\mathrm{AL}-6$ & 2 & 4 \\
\hline $\mathrm{AL}-7$ & & \\
\hline
\end{tabular}

